mit der am 1. Januar 1947 erfolgten Vereinigung der amerikanischen und britischen Zone zu einem »Gemeinsamen Wirtschaftsgebiet« wurde der sowjetischen Seite klar, dass ihre Deutschlandkonzeption - in deren Zentrum weiterhin die Reparationspolitik und das Ziel eines Einheitsstaates nach sowjetischem Vorbild standen - zum Scheitern verurteilt war. Denn aus der sogenannten Bizone entwickelte sich fortan ein deutscher Kernstaat und mithin die Integration Westdeutschlands in das von den Vereinigten Staaten dominierte westliche (europäische) System. Sichtbarster Ausdruck für die Unmöglichkeit einer gemeinsamen Besatzungspolitik bzw. für die ideologische Polarisierung der Welt in einen westlichen und östlichen Block war das Scheitern der Moskauer Außenministertagung 1947 und die Ablehnung des Marshallplanes durch die Sowjetunion. Der Plan des amerikanischen Außenministers sah bekanntlich in erster Linie vor, den europäischen Staaten inklusive der osteuropäischen Staaten ökonomische Hilfe zuteilwerden zu lassen. Für Stalin jedoch stellte die ökonomische Einbeziehung der östlichen Staaten in den amerikanischen Regierungsplan eine ernst $\mathrm{zu}$ nehmende Gefahr für den Zusammenhalt der Staaten des sowjetischen Machtbereiches dar. Gleichzeitig wurde mit dem wirtschaftlichen Aufbauplan für Europa der sowjetischen Staatsführung die eindeutige Botschaft vermittelt, dass die amerikanische Regierung keinesfalls daran dachte, sich aus Europa zurückzuziehen. ${ }^{113}$

Mit der Gründung des Kommunistischen Informationsbüros (Kominform) im September 1947, das auf eine verstärkte Kontrolle der kommunistischen Parteien abzielte und die Sowjetisierung des osteuropäischen Staatenblocks beschleunigen sollte, schritt die ideologische Formation der Welt voran. Erstmals erklärte hier der sowjetische Funktionär und Stalin-Abgesandte Andrei Schdanow die politische Leitlinie und verkündete der Weltöffentlichkeit »die These von den beiden einander in unerbittlicher Feindschaft gegenüberstehenden Lagern ${ }^{114}$

\title{
2.4 Die Genese des 'weichen Kurses in der amerikanischen Besatzungspolitik angesichts der sowjetischen Herausforderung und das endgültige Scheitern der diplomatischen Zusammenarbeit der ehemaligen Verbündeten
}

Wie gezeigt, wurde in der US-amerikanischen Administration noch während der laufenden Kriegshandlungen ein Planungsprozess in Gang gesetzt, in dem die Frage im Zentrum stand, wie nach dem militärischen Sieg mit dem »deutschen Problem« umzugehen sei. Insbesondere Präsident Roosevelt, der die Gefahren des nationalsozialistischen Expansionskrieges auch für das eigene demokratische Staatswesen sehr früh erkannte, vertrat den eindeutigen Standpunkt, dass nur eine bedingungslose Kapitulation Deutschlands im amerikanischen Interesse sei. Auch unter dem Einfluss seiner Berater stehend, kam er zu dem Entschluss, dass Deutschland kein weiteres Mal Europa mit einem Hegemonialkrieg überziehen darf. Deshalb befürworteten vor der

113 Vgl. exemplarisch zur sowjetischen Deutschlandpolitik in den ersten Nachkriegsjahren zusammenfassend Rolf Badstübner, Die sowjetische Deutschlandpolitik im Licht neuer Quellen, in: Wilfried Frage in der Nachkriegszeit, Berlin 1994, S. 102-135.

114 Cerhard Wettig, Von der Entmilitarisierung zur Aufrüstung in beiden Teilen Deutschlands 19451952, in: Bruno Thoss (Hg.), Vom Kalten Krieg zur deutschen Einheit: Analysen und Zeitzeugenberichte zur deutschen Militärgeschichte 1945 bis 1995, München 1995, S. 3-36, hier S. 8. 
Jalta-Konferenz neben dem US-Präsidenten insbesondere die einflussreichen »Roosevelt-Linken ${ }^{115}$ auf amerikanischer Seite eine Politik, die eine harte Bestrafung der Deutschen als notwendig und gerechtfertigt ansah. Obwohl in dieser Gruppe keine detaillierten Deutschlandpläne für die Zeit nach Ende des Krieges erstellt wurden Roosevelt wollte noch den endgültigen Kriegsverlauf abwarten, um nach dem Sieg über Deutschland eine festumrissene Konzeption, mit genau festgelegten Zielen, für die Besatzungspolitik auf den Weg zu bringen -, existierten hier »erste Überlegungen zur Teilung Deutschlands, zur Verurteilung der Kriegsverbrecher, Forderungen nach Reparationen, die Pflicht zum Wiederaufbau und zur Veränderung der Gesellschaftsstruktur durch die Ausschaltung der NS-Eliten und den Aufbau eines demokratischen Staatswesens « ${ }^{116}$.

Ein Produkt der Verfechter einer harten Bestrafung Deutschlands war der im Sommer 1944 in Washington entstandene berühmt gewordene sogenannte Morgenthauplan. ${ }^{117}$ Benannt nach dem Finanzminister Henry Morgenthau - der ganz unter dem Eindruck der nationalsozialistischen Massenverbrechen und insbesondere der Judenverfolgung und -ermordung stand - beinhaltete der 14 Punkte umfassende Plan unter anderem die nahezu totale Reduzierung der industriellen Produktionskapazitäten und die Umwandlung Deutschlands in ein Agrarland. Obwohl Roosevelt diesen Überlegungen durchaus Sympathien entgegenbrachte, zumal die Vorschläge Morgenthaus durchaus mit seinen Vorstellungen einer Politik der Demilitarisierung und Denazifizierung sowie der Dekartellisierung und Demokratisierung kompatibel schien, wurde der Morgenthau-Plan zu keinem Zeitpunkt ein offizielles Dokument der amerikanischen Politik. Nach heftigen Protesten von Außenminister Cordell Hull und Kriegsminister Henry Stimson wurde der Plan vom US-Präsidenten am 1. Oktober 1944 begraben. ${ }^{118}$

Gleichwohl entstand im September 1944 die vom »Geist des Morgenthau-Plans« geprägte Direktive JCS 1067, die dem künftigen amerikanischen Militärgouverneur die Richtlinien für die zu verfolgende Politik in seiner Besatzungszone in die Hand gab. Nach der Jalta-Konferenz und nach dem Tode Roosevelts im April 1945 genehmigte dessen Nachfolger Truman am 11. Mai des Jahres die Direktive. Den strafenden Charakter des Dokumentes, das die »Grundlegende[n] Ziele der Militärregierung in Deutschland « - so die Überschrift - beinhaltete, begründete Truman mit folgenden Worten: »Es muß den Deutschen klargemacht werden, daß Deutschlands rücksichtslose Kriegführung und der fanatische Widerstand der Nazis die deutsche Wirtschaft zerstört und Chaos und Leiden unvermeidlich gemacht haben und daß sie nicht der Verantwortung für das entgehen können, was sie selbst auf sich geladen haben«, so

115 Die »Roosevelt-Linken« zeichneten sich insbesondere durch die Tatsache aus, dass sie Sympathien für das sowjetische Regime unter Stalin hatten - wie beispielsweise der ehemalige Außenminister Roosevelts und von Frühjahr 1945 bis September 1946 als Handelsminister unter Truman operierende Henry Wallace, der jedwede Kritik an der Sowjetunion unterließ. Siehe hierzu und zu dem Begriff Creiner, Die Morgenthau-Legende, S. 64-67 und $72 \mathrm{ff}$.

116 Hermann-J. Rupieper, Amerikanische Besatzungspolitik, in: Benz, Deutschland unter alliierter Besatzung, S. 33-47, hier S. 33.

$117 \mathrm{Vgl}$. hierzu grundsätzlich Greiner, Die Morgenthau-Legende.

118 Benz, Potsdam 1945., S. 38. 
Truman. Unmissverständlich stellte er noch zu diesem Zeitpunkt fest: »Deutschland wird nicht besetzt zum Zwecke seiner Befreiung, sondern als besiegter Feindstaat. $^{119}$

Eine inneramerikanische Kontroverse entfachten die Vertreter des War Department und des State Department, die sich im Gegensatz zu den Anhängern des Morgenthauplans für eine "weiche Linie« in der Deutschlandfrage einsetzten. Sie stimmten einerseits mit der Ansicht überein, dass der deutsche Militarismus und der Nationalsozialismus in allen Bereichen eliminiert werden müsse, und befürworteten auch die Kontrolle des industriellen Potenzials; andererseits sah man hier die Bedeutung, Deutschland nach Kriegsende stärker in das internationale System zu integrieren und es mithin wieder in die Gemeinschaft der demokratischen Nationen aufzunehmen. Diese beiden US-Institutionen sahen in Deutschland ein Land, das fortan ein wichtiger Bestandteil eines komplexen europäischen Wirtschaftssystems sein sollte und dessen Nichteinbeziehung mit gravierenden Nachteilen für die westliche Staatengemeinschaft verbunden wäre. ${ }^{120}$

Gerade im State Department setzten früh Überlegungen ein, das postnationalsozialistische Deutschland im Interesse einer zukünftig stabilen wirtschaftlichen und politischen europäischen Friedensordnung möglichst nur mit maßvollen und erfüllbaren Bedingungen zu konfrontieren. Bereits in zwei Stellungnahmen zur Deutschlandfrage aus dem Jahre 1943 lehnte man die Teilung des Landes und radikale Eingriffe in die Wirtschaft entschieden ab und plädierte um so mehr für eine umfassende Demokratisierungspolitik, die auch und vor allem einen strukturpolitischen Umbau im föderalistischen Sinne implizierte. ${ }^{121}$

Nachdem sich Roosevelt in der Deutschlandpolitik von den genuinen Vorstellungen im Sinne Morgenthaus verabschiedet hatte, konnten allmählich die Vertreter des State Department an Einfluss gewinnen, ohne dass sich dies automatisch explizit in der konkreten amerikanischen Außenpolitik niederschlug. Das Einzige, was die Vertreter des State Departments bis zum Auftakt der Jalta-Konferenz erreichten konnten, war eine entschärfte und »dehnbarere Fassung« (Hermann Graml) der vom eindeutig harten Bestrafungscharakter Deutschlands gekennzeichneten Direktive JCS 1067. Darüber hinaus fungierte ab November 1944 mit Edward R. Stettinius als Nachfolger Hulls als Außenminister an einer zentralen Machtposition, der in der Vorbereitung auf die Konferenz in Jalta Roosevelt davon zu überzeugen versuchte, dass ein wichtiges Ziel der amerikanischen Deutschlandpolitik dereinst darin bestehen sollte, die »Eingliederung « »eines reformierten, friedlichen und wirtschaftlich nichtaggressiven Deutschlands in ein liberales Welthandelssystem « anzustreben. ${ }^{122}$ Obwohl Roosevelt in Jalta aus den bereits genannten Gründen diese Vorstellung nicht übernahm, reiste nicht Morgenthau, sondern Stettinius mit auf die Konferenz.

Nach der Jalta-Konferenz und mit der Ernennung Trumans zum neuen Präsidenten der Vereinigten Staaten verloren die Anhänger des Morgenthauplans, die nicht nur im Finanzministerium anzutreffen waren, den letzten Rest an Einfluss im Hinblick auf die amerikanische Außenpolitik. Truman gab dieser Fraktion unmissverständlich

119 Zit. n. Graml, Strukturen und Motive alliierter Besatzungspolitik in Deutschland, S. 23.

120 Siehe Rupieper, Amerikanische Besatzungspolitik, S. 34.

121 Siehe Hermann Graml, Zwischen Jalta und Potsdam, in: Vierteljahreshefte für Zeitgeschichte 24 (1976), S. 308-316, hier S. 311.

122 Loth, Die Teilung der Welt, S. 83. 
$\mathrm{zu}$ verstehen, dass sie sich in der Deutschlandfrage heraushalten sollte. Zum neuen Außenminister wählte er James F. Byrnes, dem keinerlei Sympathien für die Sowjetunion nachgesagt werden konnten und mithin in Opposition zur »Roosevelt-Linken « stand. Aber nicht nur durch den neuen Außenminister sollten sich schrittweise die veränderten Leitlinien der Deutschlandpolitik herauskristallisieren, sondern zudem auch die amerikanische Außenpolitik gegenüber der Sowjetunion. Wie gesagt, standen die Deutschlandfrage und die amerikanische Außenpolitik gegenüber der Sowjetunion nach der Potsdamer Konferenz in einem sich immer stärker aufladenden Verhältnis. Dies resultierte auf der amerikanischen Seite in allererster Linie daraus, dass Truman sich mit neuen Beratern umgab, die der mehr auf Kooperation mit Stalin angelegten Politik Roosevelts eine klare Absage erteilten. Wie beispielsweise der sich den »Riga-Grundsätzen« verpflichtende Botschafter in Moskau, W. Averell Harriman, traten sie aufgrund ihres (militanten) Antikommunismus der Politik Stalins mit Skepsis gegenüber. Eingedenk der Tatsache, dass der fundamentale Konflikt mit dem totalitären Regime der Sowjetunion ihrer Einschätzung nach während der Anti-Hitler-Koalition nur vorübergehend stillgestellt war, Stalin aber auf den alliierten Konferenzen aus einer Position der Stärke auftreten konnte - zumal als Siegermacht auf der Potsdamer Konferenz - und mithin eine nüchterne Macht- und Interessenpolitik durchzusetzen vermochte, änderte sich die amerikanische Außenpolitik. Fortan galt unter der Führung Trumans angesichts der prinzipiellen Gegnerschaft mit dem kommunistischen Sowjetsystem nach der Potsdamer Konferenz und des sich sukzessive anbahnenden Ost-West-Konfliktes die Devise: »Nicht Deutschland ist unser Problem, sondern Rußland. ${ }^{123}$

Die härtere Gangart gegenüber der Politik Moskaus zeichnete sich schon vor der Potsdamer Konferenz ab, als im April 1945 Truman gegenüber dem sowjetischen Außenminister eine Standpauke hielt, die selbst überzeugten Antikommunisten überaus bemerkenswert vorkam. Sein Ziel, dem Vertreter Moskaus die zukünftig von einer realistischen Analyse der sowjetischen Diplomatie getragene Politik Washingtons anzuzeigen, zeigte Wirkung. Obwohl Molotow den US-Präsidenten zu überzeugen versuchte, dass sich die sowjetische Regierung als zuverlässiger Partner erweisen wird, der alle getroffenen Vereinbarungen einzuhalten gedenke, musste er sichtlich beeindruckt feststellen: »Noch nie in meinem Leben hat jemand so mit mir gesprochen.« Denn angesichts der Antwort Trumans, »er solle in Zukunft seine Abmachungen einhalten, dann werde auch niemand mehr so mit ihm sprechen ${ }^{124}$, konnte er für die zu erwartende amerikanische Politik schlussfolgern: »Vorbei die Gesten, überholt die symbolischen Auftritte, mit denen Roosevelt der Anti-Hitler-Koalition immer wieder neues Leben eingehaucht hatte. «125

Auf der amerikanischen Seite gewann also unter Truman eine Politik an Boden, die im Gegensatz zum früheren Präsidenten im Hinblick auf das aus dem Zweiten Weltkrieg gestärkt hervorgegangene sowjetische System einen realistischen Blick einnahm und im Großen und Ganzen von der Prämisse ausging, dass Stalin qua kommunistischer Ideologie auf territoriale Expansion bedacht ist und sich keinesfalls zum Ziel gesetzt hatte, in grundsätzlichen Fragen mit den westlichen demokratischen

123 Zit. n. Greiner, Die Morgenthau-Legende, S. 205.

124 Loth, Die Teilung der Welt, S. 102.

125 Greiner, Die Morgenthau-Legende, S. 207. 
Staaten zusammenzuarbeiten. Dieser Wahrnehmungs- bzw. Einstellungswechsel in der US-Administration musste sich früher oder später konsequenterweise auch auf die amerikanische Deutschlandpolitik auswirken, da die Sowjetunion ihren Einflussbereich bis in die Mitte Europas, d. h. Berlin, erweitert hatte und Stalin daranging, die vagen Potsdamer Beschlüsse nach kommunistischer Lesart zu interpretieren.

Mit der Umsetzung der Direktive JCS 1067, die, wie gesagt, die Richtlinien für die Politik in der amerikanischen Besatzungszone beinhalteten, wurde das War Department beauftragt, das im Juni 1945 vom US-Präsidenten Truman die alleinige Zuständigkeit übertragen bekam. Das State Department wiederum behielt ausschließlich die Verantwortung für genuin außenpolitische Fragen, konnte gleichwohl Einfluss auf die Deutschlandpolitik nehmen, weil ihre Seite dem Militärgouverneur einen politischen Berater zur Seite stellte. Zum stellvertretenden Militärgouverneur wurde General Lucius D. Clay ernannt, der den Ermessensspielraum der Richtlinien in seinem Sinne ausnutzte und sich auch gegen Angriffe von Anhängern der »harten Linie« gegenüber Deutschland durchzusetzen wusste. Allerdings standen die allgemeinen Rahmenbedingungen der amerikanischen Besatzungszone durch die Potsdamer Beschlüsse bzw. die Direktive JCS 1067 fest. Neben grundsätzlichen politischen und gesellschaftlichen Strukturveränderungen in Deutschland fokussierte die Militärverwaltung »die >Umerziehung und >Umorientierung^der deutschen Bevölkerung in einer gewaltigen Demokratisierungsaktion auf die Werte, Einrichtungen und Spielregeln der Demokratie amerikanischer bzw. westlicher Provenienz. «126

Die umfassende Demokratisierungspolitik kam auch den Vorstellungen General Clays entgegen, der als Feind des nationalsozialistischen Gewaltsystems von der notwendigen Entnazifizierung der deutschen Gesellschaft überzeugt war. Darüber hinaus ging er aber auch davon aus, so der Hamburger Historiker Bernd Greiner, dass »die Deutschen ihren Weg zur Demokratie nur finden könnten, wenn man ihnen alsbald möglichst viel Verantwortung übertrug - in der Politik und selbstverständlich auch in der Wirtschaft. $\ll^{127}$

Die amerikanische Besatzungspolitik war spätestens seit Beginn 1946 auch durch einen Antikommunismus in der US-Administration geprägt, der sich im Laufe der Monate auch durch die Erfahrungen und Erkenntnisse über die Entwicklungen in der sowjetischen Zone - sowie durch die allmählich einsetzende Sowjetisierung der ost- und südosteuropäischen Länder - herausgebildet hatte und sich immer mehr verstärkte. »Deutschland war innerhalb weniger Monate vom Modellfall alliierter Kooperationsfähigkeit zum Testfall für den sich anbahnenden Ost-West-Konflikt geworden. $\aleph^{128}$ Nicht zuletzt das Lange Telegramm Kennans vom 22. Februar 1946 hatte einen entscheidenden Einfluss darauf, dass sich in weiten Teilen des Washingtoner Regierungsapparates ein »realistischer Blick« auf die sowjetische Außenpolitik durchsetzte. Die von ihm vorweggenommenen Prinzipien der trumanschen Eindämmungspolitik stießen insbesondere im State Department auf Resonanz, sodass sich hier gewissermaßen ein antikommunistischer Kampfgeist ausbreitete. Konsequenzen hatte dies auch für General Clay und Außenminister Byrnes, die beide keineswegs Sympathien

126 Rupieper, Amerikanische Besatzungspolitik, S. 35.

127 Greiner, Die Morgenthau-Legende, S. 259.

128 Manfred Görtemaker, Geschichte der Bundesrepublik Deutschland. Von der Gründung bis zur Gegenwart, München 1999, S. 35. 
für die Sowjetunion hatten, aber dennoch in der Deutschlandfrage durchaus die Meinung vertraten, dass zwar ein härterer Kurs gegenüber Stalin begrüßenswert wäre, dies aber nicht ausschloss, in einzelnen Fragen auf ein Arrangement zu setzen. Ab Anfang 1946 mussten die beiden einflussreichen Vertreter Washingtons indes erkennen, dass sie mit einem in Regierungskreisen weitverbreiteten Antikommunismus zu rechnen hatten und darüber hinaus auch die amerikanische Öffentlichkeit eine Appeasementpolitik gegenüber Moskau ablehnte. ${ }^{129}$

Der verstärkte Antikommunismus und die sich daraus entwickelnde Absage an einen »weichen Kurs" gegenüber Moskau mussten sich auch auf die Deutschlandpolitik der Militärregierung auswirken, sodass sich die Umsetzung der Besatzungsrichtlinien in einem politisch immer stärker aufgeladenen Kontext abspielte. Allerdings resultierten Schwierigkeiten bzw. Veränderungen in der amerikanischen Besatzungspolitik auch beispielsweise aus den wirtschaftlichen Strafmaßnahmen gegen Deutschland, wie sie die Potsdamer Beschlüsse bzw. die Direktive JCS 1067 vorsahen. Vor dem Hintergrund der 1945/46 einsetzenden Ernährungsproblematik und der Unfähigkeit der Deutschen, sich selbst zu versorgen, was unter anderem durch die Entflechtung der Industrie und durch Reparationszahlungen einschließlich der Demontagen zu erklären war, setzten aufseiten der amerikanischen Militärregierung - aber auch der britischen - Bestrebungen zu einer ökonomischen Stabilisierung des Landes ein. Denn es bestand die Gefahr, dass bei anhaltenden wirtschaftlichen und sozialen Problemen die gesamte komplexe Demokratisierungspolitik in Deutschland scheitern könnte. »Für die besatzungspolitische Praxis«, so Graml, »folgte daraus, daß sich die britischen und amerikanischen Besatzungsbehörden fast sofort daran machten, den Deutschen ihrer Zonen die Sicherung des Existenzminimums aus eigener Kraft zu ermöglichen und mithin wenigstens eine begrenzte wirtschaftliche Erholung zu fördern. ${ }^{130}$

Schon im Dezember 1945 reagierte das amerikanische Außenministerium auf das Problem der wirtschaftlichen Situation, indem es in einem Kommuniqué die Absicht bestritt, »der deutschen Wirtschaft bleibende Beschränkungen aufzuerlegen«. Vielmehr beabsichtigen die USA, »dem deutschen Volk zu gestatten [...], seine eigenen Produktionsmittel weiter $\mathrm{zu}$ entwickeln und einen höheren Lebensstandard $\mathrm{zu}$ erarbeiten«. Einschränkungen gebe es nur insofern, als es gelte, »nach Maßgabe eines späteren Friedensvertrages, die Herstellung von Waffen zu verhindern «. ${ }^{131}$

Ohne ein In-Gang-Kommen der deutschen Wirtschaft, das wesentlich die Reduzierung der Demontagen voraussetzte, wäre nicht nur der Wiederaufbau eines kapitalistischen demokratischen westeuropäischen Staatensystems unmöglich gewesen, sondern auch die allmähliche politische Integration Deutschlands in das Europa unter amerikanischer Führung. An eine einheitliche Linie unter den Alliierten war im Hinblick auf die Reparationspolitik, unter anderem aufgrund der Obstruktion der Sowjet-

129 Wie gezeigt wurde, hatte hier auch die Veröffentlichung des autobiografischen Renegatenberichtes I Chose Freedom von Krawtschenko erhebliche Bedeutung. Denn hiermit wurde die amerikanische Öffentlichkeit neuerlich darüber unterrichtet, dass die Menschen in der Sowjetunion von einem totalitären Polizeistaat regiert werden und der NKWD ein umfassendes Zwangsarbeitslagersystem mit Millionen von Häftlingen aufgebaut hatte.

130 Graml, Strukturen und Motive alliierter Besatzungspolitik in Deutschland, S. 28.

131 Görtemaker, Geschichte der Bundesrepublik Deutschland, S. 28. 
union, nicht mehr zu denken. Sollte jedoch das im Potsdamer Abkommen formulierte Ziel, Deutschland als einheitliches Wirtschaftsgebiet zu erhalten, erreicht werden, so war es angesichts des gescheiterten »Industrieplanes« - der am 28. März 1946 im Alliierten Kontrollrat vereinbart wurde - aus amerikanischer Sicht unumgänglich, in der Deutschlandpolitik einen neuen Weg einzuschlagen. Am 3. Mai 1946 verfügte General Clay einen Demontagestopp für die amerikanische Zone und problematisierte innerhalb der Militärregierung die Beziehungen zu den sowjetischen Vertretern. Wenige Monate danach verständigten sich die amerikanische und britische Militärregierung darauf, dass mit Beginn des Jahres 1947 ihre Zonen zu einem »Gemeinsamen Wirtschaftsgebiet« vereinigt werden sollten. Aus dieser Bizone entwickelte sich noch im selben Jahr, nachdem ihr auch eine politische Struktur verliehen wurde, ein westdeutscher Kernstaat. ${ }^{132}$ Das bedeutete: »Analog zur Verschärfung des Ost-West-Konfliktes zwischen der Sowjetunion und den Westmächten wurden damit nun zunehmend Maßnahmen zur Integration der beiden Teile Deutschlands in die entstehenden Machtblöcke ergriffen. «"133

Wie in Washington kam man auch in London Anfang $1946 \mathrm{zu}$ der Erkenntnis, dass das in den Potsdamer Beschlüssen formulierte Ziel, Deutschland als wirtschaftliche Einheit zu behandeln, nicht erreicht werden kann, und hielt die deutsche Teilung für wahrscheinlich. Angesichts einer sowjetischen Politik, deren strategische Absicht augenscheinlich darin lag, die politischen und ökonomischen Kosten für die Vereinigten Staaten in ihrem Deutschlandengagement so hoch wie möglich zu schrauben, um sie zum Rückzug vom europäischen Kontinent zu zwingen, änderte die englische Regierung ihre Haltung in der Deutschlandfrage. Man ging von der Annahme aus, dass Stalin mit einer großen kapitalistischen Krise Europas und Amerikas rechnete, sodass sich eine bedrohliche Konsequenz ergeben hätte, insofern die USA tatsächlich aus Europa abgezogen wären; denn dann hätte die sowjetische Supermacht alle Möglichkeiten besessen, in Europa seine politischen Vorstellungen ohne nennenswerten Widerstand durchzusetzen. Im Hinblick auf die britische Deutschlandpolitik hieß es in einem Arbeitspapier vom 5. April 1946 eindeutig: »[U]nsere langfristige Politik in bezug auf Deutschland muß in vollem Umfang der Tatsache Rechnung tragen, daß Rußland ein sehr viel gefährlicherer potenzieller Feind ist als Deutschland; sie kann deshalb nicht allein unter dem Gesichtspunkt gestaltet werden, ein Wiederaufleben der Bedrohung durch Deutschland zu verhindern. «134

Kurze Zeit später, am 3. Mai 1946, bezog der englische Außenminister Ernest Bevin eine richtungsweisende Position, als er in einer Kabinettsvorlage feststellte, dass die Gefahr, die seitens der sowjetischen Politik drohte, »inzwischen mit Sicherheit genauso groß [sei], möglicherweise noch größer, als die Gefahr eines wiedererstarkten Deutschland «. ${ }^{135}$ Er zog daraus den Schluss, dass die Westzonen - ohne Rücksicht auf sowjetische Störmanöver - innerhalb kürzester Zeit wiederaufgebaut werden sollten. ${ }^{136}$

132 Dülffer, Jalta, 4. Februar 1945, S. 210.

133 Görtemaker, Geschichte der Bundesrepublik Deutschland, S. 38.

134 Zit. n. ebd., S. 36.

135 Zit. n. ebd.

136 Ebd. 
Die britische und die amerikanische Regierung erkannten also die Notwendigkeit, angesichts der sowjetischen Herausforderung in Zukunft mit einer einheitlicheren Politik der westlichen demokratischen Staaten zu antworten. Dies zeitigte selbstverständlich, wie bereits angesprochen wurde, Konsequenzen in Bezug auf die Deutschlandkonzeptionen der beiden alliierten Partner, zumal sich in diesem Kontext die Differenzen zur Sowjetunion immer mehr verstärkten. Eine Weiche für die Einrichtung der Bizone und die Teilung Deutschlands wurde durch das Scheitern der Pariser Außenministerkonferenz im Frühjahr und im Sommer 1946 gestellt. Ein Kompromiss in den vom Außenminister Molotow vorgelegten Moskauer Forderungen war unmöglich. Denn die sahen unter anderem die Umstrukturierung der deutschen Wirtschaft auf der Grundlage der in der sowjetischen Besatzungszone vollzogenen Praxis vor, und das hätte für die Zukunft die kommunistische Umgestaltung Westdeutschlands impliziert. Vor dem Hintergrund der beabsichtigten Integration der westlichen Besatzungszonen in den eigenen Machtbereich hielt der amerikanische Außenminister Byrnes am 6. September 1946 seine richtungsweisende programmatische Rede in Stuttgart. Neben der Ankündigung der Einrichtung der Bizone, die wie gesagt im Kern auf die Bildung eines Weststaates und mithin auf die Teilung des Landes hinauslief, sorgte insbesondere die Bekanntgabe, dass die Vereinigten Staaten ihre militärische Präsenz in Deutschland und in Europa solange aufrechterhalten lassen, wie es andere Mächte auch tun. Für die deutsche Bevölkerung bedeutete die Ankündigung der fortdauernden Anwesenheit der US-Truppen auch den Schutz vor der als bedrohlich empfundenen sowjetischen Expansion. ${ }^{137}$

Das Scheitern der Moskauer Außenministerkonferenz im März und April 1947 bestätigte nur die frühe Einsicht von Teilen des Washingtoner Regierungsapparates vor allem vom State Departement-, dass eine substanzielle Zusammenarbeit mit Moskau beim Wiederaufbau Europas unmöglich sei und die Deutschlandfrage in der sich abzeichnenden Ost-West-Blockkonfrontation politische Brisanz enthielt. Vor dem Hintergrund der ökonomischen Krise Europas und des Ziels, Deutschland in die westliche Allianz einzubinden, kam es im Laufe des Jahres 1947 unter Truman mit Blick auf die gegenüber der Sowjetunion zu vertretende Politik zu zentralen Veränderungen. Nachdem er im März seine »Eindämmungsdoktrin« verkündet hatte und erstmals öffentlich von der Zweiteilung der Welt in ein westlich-demokratisches sowie östlichkommunistisches Lager gesprochen hatte, kündigte drei Monate darauf der neue USAußenminister General George C. Marshall einen neuen Kurs an. Am 5. Juni 1947 sagte der Nachfolger von Byrnes - ausgehend von der Prämisse, dass sich der sowjetische Expansionsdrang global manifestiere und mithin auch global eingedämmt werden müsse - in einer programmatischen Rede vor Absolventen der Harvard-Universität:

Es ist nur logisch, daß die Vereinigten Staaten alles tun, was in ihrer Macht steht, um die Wiederherstellung gesunder wirtschaftlicher Verhältnisse in der Welt zu fördern, ohne die es keine politische Stabilität und keinen sicheren Frieden geben kann. Unsere Politik richtet sich nicht gegen irgendein Land oder irgendeine Doktrin, sondern gegen Hunger, Armut, Verzweiflung und Chaos. Ihr Zweck ist die Wiederbelebung einer funk- 
tionierenden Weltwirtschaft, damit die Entstehung politischer und sozialer Bedingungen ermöglicht wird, unter denen freie Institutionen existieren können. ${ }^{138}$

Gleichwohl waren sich der amerikanische Außenminister und seine einflussreichen politischen Berater, zu denen beispielsweise der ehemalige Mitarbeiter in der Moskauer Botschaft, Chip Bohlen, und der Leiter des im Frühjahr 1947 eingerichteten Policy Planning Staff, George F. Kennan, zählten, darüber im Klaren, dass sich der entscheidende »Kalte-Kriegs-Schauplatz« in Europa befindet. Der am 3. April 1948 vom Kongress verabschiedete Marshallplan versprach Deutschland und allen anderen europäischen Ländern umfangreiche finanzielle Hilfe. Für die Verwaltung der Gelder in Europa war der Leiter der in Paris sitzenden Organisation für wirtschaftliche Zusammenarbeit in Europa, Averell Harriman, ${ }^{139}$ zuständig. Im Kontext der Containmentpolitik hatte der Marshallplan neben der wirtschaftlichen Strategie im Hinblick auf die Eindämmung der ideologischen und machtpolitischen Expansion des Sowjetkommunismus auch eine explizit politische Bedeutung. Denn durch den ökonomischen Wiederaufbau Westeuropas sollten auch die Voraussetzungen geschaffen werden, um demokratische Verhältnisse zu verwirklichen, die wiederum als Garantie angesehen wurden, die westeuropäischen Staaten gegen kommunistische Einflüsse zu immunisieren; in diesem Zusammenhang flossen auch finanzielle Mittel der CIA über vertrauliche Fonds in den Marshallplan, die in kulturelle Projekte investiert wurden, um die Werte des Westens gegenüber der kommunistischen Herausforderung »offensiv« zu vertreten.

Auf Vorschlag von Kennan, dem eigentlichen Architekten für das European Recovery Program (ERP), ${ }^{140}$ wurde aus taktischen Gründen auch der Sowjetunion und den Ländern seines Einflussbereiches Unterstützung angeboten. Wie erwartet, mussten

138 Zit. n. der deutschen Übersetzung in: Europa-Archiv 2 (1947), S. 821.

139 Es ist alles andere als ein Zufall, dass Averell Harriman im Kontext des Kalten Krieges mit der Koordination des Marshallplans als Leiter der Organization for European Economic Cooperation beauftragt wurde. Wie gezeigt, war Harriman ein führender Repräsentant in der US-Administration, der frühzeitig eine antikommunistische Haltung einnahm. Schon zur Zeit seiner Tätigkeit als amerikanischer Botschafter in Moskau während des Zweiten Weltkrieges riet Harriman - z. B. auf der Jalta-Konfernz - auch zu einer harten Haltung gegenüber der Sowjetunion. Eingedenk der Erkenntnisse über das kommunistische Regime unter Stalin misstraute er jedweder Appeasementpolitik. Frühzeitig verfestigte sich bei ihm der Eindruck, dass der totalitäre Diktator ein fundamental anderes Weltbild vertritt und sich nach amerikanischer Auffassung zu diamentral gegensätzlichen Werten und Prinzipien bekennt. Nach Kriegsende rechnete Harriman mit einem sowjetischen Vormachtstreben in Europa und sah die Implikationen für die amerikanische Deutschlandpolitik. Als Nachfolger von Henry Wallace als Handelsminister im Herbst 1946 unter Präsident Truman wandte er sich entschieden gegen die harte Bestrafung Deutschlands, wie es der Morgenthauplan vorsah. Angesichts der kommunistischen Cefahr plädierte er für die Einbindung (West-)Deutschlands in die westliche Allianz. Interessanterweise entschied sich Harriman während der Tätigkeit als US-Botschafter in Moskau im Frühjahr 1944 dazu, Kennan als Mitarbeiter zu gewinnen, um u. a. die »Russlandpolitik« der Regierung zu erörtern. So entstanden noch im Verlauf des Krieges gegen Deutschland unter der Leitung Harrimans einige Abhandlungen des Russlandexperten Kennan, die als Vorarbeiten für die Theorie des Containment angesehen werden können (siehe Kennan, Memoiren eines Diplomaten, S. 251-256).

140 Siehe hierzu die Ausführungen in dem Kapitel »Der Marshallplan« in der Autobiografie Kennans, ebd., S. 328-356. 
dann im Sommer 1947 einige Länder Osteuropas das Angebot des amerikanischen Außenministers ablehnen, nachdem ihnen Stalin - wie zum Beispiel im Fall der Tschechoslowakei und Polens - mit ernsthaften Konsequenzen gedroht hatte.

Wie gezeigt, hatte sich aus der Wahrnehmungsperspektive des Kremlchefs die Welt durch den Marshallplan in zwei unversöhnliche Lager gespalten: in ein unter der Führung der USA stehendes "imperialistisches und antidemokratisches Lager « auf der einen Seite und in ein "antiimperialistisches und demokratisches Lager unter der Führung der Sowjetunion auf der anderen Seite. Diese im September 1947 auf der Gründungskonferenz der Kominform vom ZK-Sekretär Schdanow in Stalins Auftrag verkündete »Zwei-Lager-Theorie« war fortan für sämtliche kommunistische Parteien verbindlich. Zudem zeigte sie unmissverständlich an, dass Stalin der Zusammenarbeit mit den alliierten Partnern ganz offiziell ein Ende gesetzt hatte und der alten Linie, d.h. dem ideologischen Grundsatzkonflikt mit den kapitalistischen Staaten, absolute Priorität einräumte.

Die sowjetische Ablehnung des Marshallplanes war die entscheidende Weichenstellung für die Spaltung Europas und Deutschlands, da sie den Ausbau der Bizone, die Einführung der Währungsreform und die Gründung eines separaten Weststaates beschleunigte. In der Konzeption des amerikanischen Außenministers Marshall besaß naheliegenderweise Westdeutschland respektive die westlichen Besatzungszonen eine Schlüsselfunktion. Mithin erzwang das europäische Wiederaufbauprogramm eine veränderte amerikanische Deutschlandpolitik, was sich in der neuen Besatzungsdirektive JCS 1779 niederschlug, die im Juli 1947 die alte JCS 1067 ablöste. Bereits in seiner Harvard-Rede hatte Marshall bekräftigt, »in Deutschland jene politischen, wirtschaftlichen und moralischen Verhältnisse zu schaffen, die am effektivsten zu einem stabilen und prosperierenden Europa beitragen $\ll^{141}$. Und auch der innerhalb der Truman-Administration über sehr viel Einfluss verfügende Harriman konstatierte in Bezug auf die Bedeutung der westlichen Besatzungszonen für den Wiederaufbau Europas: »Wir können nicht eine sich selbst tragende westeuropäische Wirtschaft auf die Beine stellen ohne ein gesundes Deutschland. ${ }^{142}$

Die in JCS 1779 neuformulierte Deutschlandpolitik stellte eine Zäsur dar. Im Hinblick auf die Integration der Deutschen in das westliche Staatenbündnis sah die zukünftige amerikanische Besatzungspolitik vor, die durch die alte Direktive JCS 1067 resultierenden Behinderungen in der Entmilitarisierung, Entkartellisierung und Entnazifizierung zu beseitigen. Beispielsweise führte das dazu, dass die nach dem Krieg in Gang gesetzten politischen Säuberungen mehr und mehr an Schwung verloren, da sie für die desaströse wirtschaftliche Situation in den westlichen Zonen mitverantwortlich gemacht wurden. ${ }^{143}$

Mit dem Marshallplan übernahmen die Vereinigten Staaten die Führungsrolle in Europa. In der existenziellen Systemauseinandersetzung mit dem sowjetischen Kommunismus wurde hierbei die Notwendigkeit gesehen, nur knapp zwei Jahre nach dem Sieg über den Nationalsozialismus (West-)Deutschland zum Partner zu machen. Entscheidend war der Gedanke, dass dem Kommunismus am effektivsten Paroli geboten werden kann, wenn der wirtschaftliche Wiederaufbau Europas in Gang gesetzt

141 Zit. n. Greiner, Die Morgenthau-Legende, S. 327.

142 Zit. n. ebd., S. 331.

143 Vgl. Rupieper, Amerikanische Besatzungspolitik, S. 46. 
werden kann. Der Marshallplan beschleunigte die Spaltung Europas und Deutschlands und führte zur Integration des westlichen Landesteiles in die westliche Allianz. Hier im Zentrum Europas eskalierte im Verlauf des Jahres der Konflikt zwischen dem westlichen System unter der Führung der USA und dem östlichen System unter der Führung der Sowjetunion. In der Berlin-Krise im Jahre 1948 spitzte sich die Ost-WestBlockkonfrontation $\mathrm{zu}$ und bedeutete die erste große politische Krise zwischen den beiden Supermächten.

\section{Berlin als Schnittpunkt der feindlichen Systeme und die Krise 1948/49}

Die Verschärfung des Ost-West-Gegensatzes wirkte sich freilich auch auf die gemeinsame alliierte Besatzungspolitik in Berlin aus und spitzte sich nach der Einführung der getrennten Währungsreform im Westen und Osten Deutschlands 1948 mit der Blockade der Stadt durch die Sowjetunion dramatisch zu. Vor dem Hintergrund, dass Berlin die ehemalige Reichshauptstadt des nationalsozialistischen Regimes war und sozusagen das Machtzentrum des »Führerstaates« darstellte, wo zentrale Entscheidungen im Eroberungs- und Vernichtungskrieges getroffen wurden, besaß die Stadt einen hohen symbolischen Stellenwert für die alliierten Siegermächte. Noch vor der bedingungslosen Kapitulation des >Dritten Reiches`am 8. Mai 1945 hatte Berlin durch die Londoner European Advisory Commission einen Sonderstatus erhalten. Das »Protokoll über die Besatzungszonen in Deutschland und die Verwaltung von Berlin« (Londoner Protokoll) vom 12. September 1944 diente als Grundlage für die konkreten Abmachungen der Konferenzen von Jalta und Potsdam. Die Alliierten planten, in Berlin eine gemeinsame Besatzungsherrschaft ausüben zu wollen. Dazu wurde die Stadt in drei Sektoren eingeteilt, d. h. in eine amerikanische, britische und sowjetische Besatzungszone; später entstand aus zwei Sektoren der Briten die französische Zone. $\mathrm{Zu}$ diesem Zeitpunkt lebten schätzungsweise 1,8 Millionen Menschen in den westlichen Sektoren, im östlichen etwa 1,1 Millionen.

In einem weiteren Abkommen vom 14. November 1944 wurde vereinbart, einen Alliierten Kontrollrat ins Leben zu rufen, der als die entscheidende gemeinsame Behörde ihren Sitz in Berlin haben sollte. Die gemeinsame Verwaltung und Regierung der Stadt lag in den Händen der Alliierten Kommandantur, einer Art »Kontrollrat en miniature (Wolfgang Benz), die aus den vier Oberbefehlshabern der alliierten Truppen bestand, dem Alliierten Kontrollrat direkt unterstand und am 11. Juli 1945 seine Arbeit aufnahm. Damit war die Anwesenheit der vier Siegermächte im Zentrum der Stadt festgelegt, von dem aus das Nachkriegsdeutschland gemeinschaftlich regiert werden sollte. Hierbei wurde die gemeinsame Zusammenarbeit in der Kommandaturverwaltung von Anbeginn durch die Möglichkeit der einzelnen Stadtkommandanten infrage gestellt, letzten Endes - insofern bei einzelnen Problemen kein gemeinsamer Beschluss gefasst wurde - selbstständig in den jeweiligen Zonen zu operieren. Je mehr sich indes das Verhältnis der Alliierten insgesamt verschlechterte und die Deutschlandfrage an Brisanz gewann, umso größer wurde die Bedeutung der einzelnen Militärregierungen. ${ }^{144}$ 